\title{
A double rotor coherent state construction of the Wigner supermultiplet $\mathrm{SU}(4) \supset \mathrm{SU}(2) \times \mathrm{SU}(2)$
}

\author{
K T Hecht \\ Physics Department, University of Michigan, Ann Arbor, MI 48109, USA
}

Received 28 September 1993

\begin{abstract}
A double rotor vector coherent state construction of the SU(4) $\supset S U(2) \times S U(2)$ Wigner supermultiplet leads to a simple matrix representation of this scheme. Matrix elements of the vector coherent state realizations of the group generators are given by very simple expressions in terms of ordinary spin $(S)$ and isospin $(T)$ Wigner coefficients with intrinsic projection labeis $K_{S}$ and $K_{T}$. The $\mathcal{K}$-matrix technique is used to effectively elevate these labels to the status of good quantum numbers. The $\mathcal{K K}^{\dagger}$ matrices are given in analytic form for a number of important irreducible representations.
\end{abstract}

\section{Introduction}

Vector coherent state (VCS) theory $[1,2]$ has now been used to give very explicit expressions for the matrix representations of many higher-rank symmetry algebras of interest in applications to physical problems [3]. The vector coherent state construction is an induction process in which a representation of a simple subalgebra (or subgroup) is augmented to a representation of a larger full algebra (or group). Two types of vcs expansions have been used to great advantage in this construction process. The most commonly used vCS construction involves a boson expansion in terms of a set of $n$ Bargmann variables, $z$, leading to a set of orthonormal basis functions with a scalar product defined in terms of the standard exponential Bargmann measure. In a more recent development [4], vCS theory has been used to generate rotor expansions in terms of standard angular variables, making use of the conventional angular measure of angular momentum coherent state theory. In particular, Rowe, Le Blanc and Repka [4] have used a coherent state rotor expansion to give a simple yet powerful construction of the matrix representations of the $S U(3) \supset S O(3)$ basis of good orbital angular momentum. In this construction the projection label, $K$, of the Elliott angular momentum projection technique [5] has effectively been elevated to the status of a good quantum number. Despite the power of the rotor coherent state expansion, the $\mathrm{SU}(3) \supset \mathrm{SO}(3)$ group chain is so far the only example for which this technique has been fully exploited $[6,7] \dagger$.

It is the purpose of this contribution to show that there is a second example, also with well known nuclear structure applications, in which a coherent state rotor expansion leads to a simple but elegant construction of irreducible representations of a higher rank group, namely the $S U(4) \supset S U(2) \times S U(2)$ Wigner supermultiplet group. A complete labelling scheme for this group has been achieved by Draayer [8] who used the Elliott angular

$\dagger$ See note added in proof. 
momentum projection technique to augment the spin and isospin quantum numbers $\left(S M_{S}\right)$, $\left(T M_{T}\right)$ with the projection labels $K_{S}$ and $K_{T}$. In order to calculate the generator matrix elements and SU(4) reduced Wigner coefficients in this fully labelled but non-orthonormal basis, Draayer calculates the transformation coefficients from the $K_{S}\left(S M_{S}\right), K_{T}\left(T M_{T}\right)$ basis to the canonical, fully specified orthonormal $U(4) \supset U(3) \supset U(2) \supset U(1)$ basis. Since the resultant calculational algorithm is somewhat laborious, this method has not been widely used in actual applications. To date, the only analytical expressions known for SU(4) reduced Wigner coefficients in the supermultiplet scheme are those for the special multiplicity-free irreducible representations for which the basis states are fully specified by $S M_{S}, T M_{T}$ alone [9]. A coherent state rotor expansion of the Rowe-Le Blanc-Repka type can again be used to convert the labels $K_{S}, K_{T}$ of the Draayer scheme into good quantum numbers through the $\mathcal{K}$-matrix theory utilized in all vCS constructions. vCS representations are in general non-unitary or Dyson representations. The $\mathcal{K}$-matrix transformation gives a systematic and simple algorithm for transforming the vCS matrices into unitary form. In addition, the zeros of the eigenvalues of the $\mathcal{K} \mathcal{K}^{\dagger}$ matrix define the physical subspace of the vCS basis. The possible $K_{S}, K_{T}$ values together with the zeros of the $\mathcal{K} \mathcal{K}^{\dagger}$ eigenvalues thus also give a very simple method of determining the multiplicity of the possible $S, T$ values in a given SU(4) irreducible representation. Due to the central role of the $\mathcal{K}$ matrices, it will be shown with numerous examples in section 3 that these can be calculated in general analytical form. Section 2 gives the double rotor coherent state realization of the $\mathrm{SU}(4) \supset \mathrm{SU}(2) \times \mathrm{SU}(2)$ generators. Together with the $\mathcal{K}$ matrices of section 3, these give a simple direct evaluation of the SU(4) generator matrix elements which can lead to a full determination of the Wigner-Racah calculus for the $S U(4) \supset S U(2) \times S U(2)$ supermultiplet scheme.

\section{The double rotor expansion of the $S U(4) \supset S U(2) \times S U(2)$ algebra}

The supermultiplet scheme is based on the four spin-charge states of a single nucleon, $\left|m_{s} m_{t}\right\rangle$, with

$$
\begin{array}{ll}
|a\rangle=\left|+\frac{1}{2}+\frac{1}{2}\right\rangle & |b\rangle=\left|-\frac{1}{2}-\frac{1}{2}\right\rangle \\
|c\rangle=\left|+\frac{1}{2}-\frac{1}{2}\right\rangle & |d\rangle=\left|-\frac{1}{2}+\frac{1}{2}\right\rangle .
\end{array}
$$

To gain the most convenient double rotor expansion it will be useful to define the basis states $|i\rangle, i=1, \ldots, 4$, by

$$
\begin{array}{rlrl}
|a\rangle & =\frac{1}{\sqrt{2}}(|1\rangle+|2\rangle) & |b\rangle & =\frac{1}{\sqrt{2}}(-|1\rangle+|2\rangle) \\
|c\rangle=\frac{1}{\sqrt{2}}(|3\rangle+|4\rangle) & |d\rangle=\frac{1}{\sqrt{2}}(-|3\rangle+|4\rangle)
\end{array}
$$

and define the 15 supermultiplet generators [9], $S, T$, and $E$, in terms of U(4) generators, $C_{i j}$,

$$
C_{i j}=\sum_{\alpha} a_{\alpha t}^{\dagger} a_{\alpha j} \quad i, j=1, \ldots, 4
$$

where $i, j$ give the spin, isospin quantum numbers and $\alpha$ stands for all additional (orbital) quantum numbers needed to specify the single-nucleon creation and annihilation operators. 
In terms of the $C_{i j}$ the generators are

$$
\begin{aligned}
& S_{0}=\frac{1}{2}\left(C_{12}+C_{21}+C_{34}+C_{43}\right) \\
& S_{+}=\frac{1}{2}\left(-C_{13}-C_{23}+C_{14}+C_{24}-C_{31}+C_{32}-C_{41}+C_{42}\right) \\
& S_{-}=\frac{1}{2}\left(-C_{31}-C_{32}+C_{41}+C_{42}-C_{13}+C_{23}-C_{14}+C_{24}\right) \\
& T_{0}=\frac{1}{2}\left(C_{12}+C_{21}-C_{34}-C_{43}\right) \\
& T_{+}=\frac{1}{2}\left(C_{13}+C_{23}+C_{14}+C_{24}+C_{31}-C_{32}-C_{41}+C_{42}\right) \\
& T_{-}=\frac{1}{2}\left(C_{31}+C_{32}+C_{41}+C_{42}+C_{13}-C_{23}-C_{14}+C_{24}\right) \\
& E_{00}=\frac{1}{2}\left(C_{11}+C_{22}-C_{33}-C_{44}\right) \\
& E_{10}=\frac{1}{2 \sqrt{2}}\left(C_{13}+C_{23}-C_{14}-C_{24}-C_{31}+C_{32}-C_{41}+C_{42}\right) \\
& E_{-10}=\frac{1}{2 \sqrt{2}}\left(-C_{31}-C_{32}+C_{41}+C_{42}+C_{13}-C_{23}+C_{14}-C_{24}\right) \\
& E_{01}=\frac{1}{2 \sqrt{2}}\left(-C_{13}-C_{23}-C_{14}-C_{24}+C_{31}-C_{32}+C_{42}-C_{41}\right) \\
& E_{0-1}=\frac{1}{2 \sqrt{2}}\left(C_{31}+C_{32}+C_{41}+C_{42}-C_{13}+C_{23}-C_{24}+C_{14}\right) \\
& E_{11}=\frac{1}{2}\left(-C_{11}+C_{22}+C_{12}-C_{21}\right) \\
& E_{-1-1}=\frac{1}{2}\left(-C_{11}+C_{22}-C_{12}+C_{21}\right) \\
& E_{1-1}=\frac{1}{2}\left(C_{33}-C_{44}-C_{34}+C_{43}\right) \\
& E_{-11}=\frac{1}{2}\left(C_{33}-C_{44}+C_{34}-C_{43}\right) .
\end{aligned}
$$

The phases and normalizations of the $E_{\alpha \beta}$ are chosen to put these into standard double spherical tensor form, with $E_{\alpha \beta}=T_{M_{S}=\alpha, M_{T}=\beta}^{S=1, T=1}$. The SU(4) irreducible representations are labelled by four-rowed Young tableaux partition labels $\left[f_{1}, f_{2}, f_{3}, f_{4}\right]$, by the SU(4) labels $\left\{\lambda_{1}, \lambda_{2}, \lambda_{3}\right\}$, or by the Wigner supermultiplet (or standard Cartan $\operatorname{SO}(6)$ labels $\left(P, P^{\prime}, P^{\prime \prime}\right)$ ) with

$$
\begin{aligned}
& \lambda_{1}=f_{1}-f_{2} \quad \lambda_{2}=f_{2}-f_{3} \quad \lambda_{3}=f_{3}-f_{4} \\
& P=\frac{1}{2}\left(\lambda_{1}+2 \lambda_{2}+\lambda_{3}\right) \quad P^{\prime}=\frac{1}{2}\left(\lambda_{1}+\lambda_{3}\right) \quad P^{\prime \prime}=\frac{1}{2}\left(\lambda_{1}-\lambda_{3}\right) .
\end{aligned}
$$

These characterize the highest-weight state $|\phi\rangle$ with

$$
\begin{aligned}
& C_{i j}|\phi\rangle=0 \quad \text { for } \quad i<j \\
& C_{11}|\phi\rangle=\left(\lambda_{1}+\lambda_{2}+\lambda_{3}\right)|\phi\rangle \quad C_{22}|\phi\rangle=\left(\lambda_{2}+\lambda_{3}\right)|\phi\rangle \\
& C_{33}|\phi\rangle=\lambda_{3}|\phi\rangle \quad C_{44}|\phi\rangle=0 .
\end{aligned}
$$

The double rotor expansion uses the double rotation operator $R(\Omega) \equiv R\left(\Omega_{S}\right) R\left(\Omega_{T}\right)$, with Euler angles $\alpha_{S}, \beta_{S}, \gamma_{S} \equiv \Omega_{S}$ and $\alpha_{T}, \beta_{T}, \gamma_{T} \equiv \Omega_{T}$ in the spin and isospin space. Draayer [8] has shown that the set of states, $\{R(\Omega)|\phi\rangle\}$, obtained by rotation of the highestweight state through all possible angles $\alpha_{S}, \ldots, \gamma_{T}$, spans the full SU(4) space. Arbitrary state vectors $|\Psi\rangle$ in this space are now tranformed into their coherent state realizations with a coherent state wavefunction

$$
\Psi(\Omega)=\langle\phi|R(\Omega)| \Psi\rangle .
$$

A state $\left|\alpha S M_{S} T M_{T}\right\rangle$ with definite spin and isospin quantum numbers is represented by

$$
\begin{aligned}
\Psi_{\alpha S M_{S} T M_{T}}(\Omega) & =\left\langle\phi|R(\Omega)| \alpha S M_{S} T M_{T}\right\rangle \\
& =\sum_{K_{S}, K_{T}}\left\{\phi\left|\alpha S K_{S} T K_{T}\right\rangle D_{K_{S} M_{S}}^{S}\left(\Omega_{S}\right) D_{K_{T} M_{T}}^{T}\left(\Omega_{T}\right) .\right.
\end{aligned}
$$


The convention to be used for the rotation operators $R(\Omega)$ and the $S U(2) D$-functions will be those of Rowe et al [4]. Since these are different from the 'conventional' nuclear physics choices $[5,8,10]$ (note in particular the interchange of the role of $K$ and $M$ quantum numbers relative to the common nuclear conventions [10]), they will be carefully defined in the appendix. The work of Draayer [8] shows that the SU(4) irreducible representations $\left[f_{1}, f_{2}, f_{3}, f_{4}\right] \equiv\left\{\lambda_{1} \lambda_{2} \lambda_{3}\right\}$ are spanned by the double rotor wavefunctions with $K_{S}, K_{r}$ values restricted by

$$
\begin{aligned}
& \left(K_{S}+K_{T}\right)= \pm \lambda_{1}, \pm\left(\lambda_{1}-2\right), \pm\left(\lambda_{1}-4\right), \ldots, 0 \text { (or } \pm 1 \text { ) } \\
& \left(K_{S}-K_{T}\right)= \pm \lambda_{3}, \pm\left(\lambda_{3}-2\right), \pm\left(\lambda_{3}-4\right), \ldots, 0 \text { (or } \pm 1 \text { ). }
\end{aligned}
$$

The double rotor realizations of the SU(4) generators of this section will show that the SU(4) double rotor coherent state wavefunctions are spanned by the symmetrized (normalized) double rotor functions

$$
\begin{aligned}
\Psi_{K_{S} K_{T}: S M_{S} T M_{T}}(\Omega) & =\frac{1}{8 \pi^{2}}\left[\frac{(2 S+1)(2 T+1)}{2\left(1+\delta_{K_{S} 0} \delta_{K_{\mathrm{T}} 0}\right)}\right]^{\frac{1}{2}} \\
& \times\left\{D_{K_{S} M_{S}}^{S}\left(\Omega_{S}\right) D_{K_{T} M_{T}}^{T}\left(\Omega_{T}\right)+(-1)^{\lambda_{2}+\lambda_{3}+S+T} D_{-K_{S} M_{S}}^{S}\left(\Omega_{S}\right) D_{-K_{T} M_{T}}^{T}\left(\Omega_{T}\right)\right\} .
\end{aligned}
$$

To specify the double rotor $\Psi_{K_{S} K_{T}}$ it will thus be sufficient to choose $K_{S} \geqslant 0$, and for $K_{S}=0: K_{T} \geqslant 0$. (The phase factor $(-1)^{\lambda_{2}+\lambda_{3}+S+T}$ in the symmetrized functions of equation (10) will be established below.) The requirement $S \geqslant\left|K_{S}\right|, T \geqslant\left|K_{T}\right|$, together with the structure of the $\mathcal{K} \mathcal{K}^{\dagger}$ matrices (section 3), will thus determine the multiplicity of a given $S, T$ value. For states with low values of $S+T$, for which the eigenvalues of $\mathcal{K} \mathcal{K}^{\dagger}$ are all non-zero (no redundant states), the number of occurrences of a given $S, T$ will be determined by the number of possible $K_{S}, K_{T}$ combinations. The maximum possible value of $S+T$ is $S+T=\lambda_{1}+\lambda_{2}+\lambda_{3}=f_{1}-f_{4}$, and, for this maximum $S+T$ value, $S_{\min }$ or $T_{\min }$ are given by $\frac{1}{2}\left(\lambda_{1}+\lambda_{3}\right)$ [8]. States with $S+T=\lambda_{1}+\lambda_{2}+\lambda_{3}$, with $S($ or $T) \geqslant \frac{1}{2}\left(\lambda_{1}+\lambda_{3}\right)$, always have an occurrence of 1 . For these $S, T$ values the $\mathcal{K} \mathcal{K}^{\dagger}$ matrix always has only a single non-zero eigenvalue giving only a single non-redundant or physically allowed state. In general, the states with $S+T \geqslant \lambda_{2}+2$ will have $\mathcal{K} \mathcal{K}^{\dagger}$ matrices with some zero eigenvalues and hence some physically forbidden states. Table 1 gives a specific example-the possible $S, T$ values for the irreducible representation [8620] with $\left\{\lambda_{1} \lambda_{2} \lambda_{3}\right\}=\{242\}$. In this case there are five possible symmetrized states of the type of equation (10), with $K_{S} K_{T}=20,11,1-1,02$ and 00 . Note that states with $K_{S} K_{T}=00$ must have $S+T=$ even since $\lambda_{2}+\lambda_{3}=$ even. States with both $S$ and $T \geqslant 2$ can thus have a 5 -fold occurrence for $S+T=$ even and a 4 -fold occurrence for $S+T=$ odd. The maximum $S+T$ value is 8 in this case. States with $S+T=8, S($ or $T$ ) $\geqslant 2$, are all single. The $\mathcal{K} \mathcal{K}^{\dagger}$ matrix for this case has four zero eigenvalues. In addition, it can be shown (section 3) that the $\mathcal{K} \mathcal{K}^{\dagger}$ matrices for states with $S+T=7$ have two zero eigenvalues, thus reducing the possible number of physical states by two, while states with $S+T=6$ lead to $\mathcal{K} \mathcal{K}^{\dagger}$ matrices with one zero eigenvalue, reducing the possible number of physical states by one. Note also that the $S T$ value 00 can occur only in irreducible representations with $\lambda_{1}$ and $\lambda_{3}$ both even (so that $K_{S} K_{T}=00$ is possible; the additional requirement $\lambda_{2}+S+T=$ even further requires $\lambda_{2}=$ even). For irreps with $\lambda_{1}, \lambda_{3}=$ odd, odd; or $\lambda_{1}, \lambda_{3}=$ even, even, but $\lambda_{2}=$ odd; the minimum $S T$ values are 10 or 01 . For irreps with $\lambda_{1}, \lambda_{3}=$ even, odd or odd, even the minimum $S T$ value is always $\frac{1}{2} \frac{1}{2}$. These minimum $S T$ values are always single (with an occurrence of 1 ). 
Table 1. The possible $S T$ values for the irrep [8620].

\begin{tabular}{|c|c|c|c|c|c|c|c|}
\hline \multirow[b]{3}{*}{$(62)^{l}$} & & $(60)^{1}$ & & & & \multirow{4}{*}{$(20)^{2}$} & \multirow{9}{*}{$(00)^{1}$} \\
\hline & $(61)^{1}$ & & $(50)^{1}$ & & & & \\
\hline & $(52)^{2}$ & & $(41)^{3}$ & & $(30) 1$ & & \\
\hline$(53)^{1}$ & & $(42)^{4}$ & & $(31)^{4}$ & & & \\
\hline (44) & $(43)^{2}$ & $(33)^{4}$ & & $(22)^{5}$ & $(21)^{3}$ & $(11)^{3}$ & \\
\hline & $(34)^{2}$ & & $(23)^{4}$ & & $(12)^{3}$ & & \\
\hline$(35)^{\prime}$ & $(25)^{2}$ & $(24)^{4}$ & $(14)^{3}$ & $(13)^{4}$ & $(03)^{1}$ & $(02)^{2}$ & \\
\hline$(26)^{1}$ & & $(15)^{3}$ & & $(04)^{2}$ & & & \\
\hline & $(16)^{1}$ & $(06)^{1}$ & $(05)^{1}$ & & & & \\
\hline
\end{tabular}

In the VCS, rotor expansion operators $X$ are transformed into their vCS realizations, $\Gamma(X)$, through $X|\Psi\rangle \rightarrow \Gamma(X) \Psi(\Omega)$, with (cf equation (7))

$$
\Gamma(X) \Psi(\Omega)=\langle\phi|R(\Omega) X| \Psi\rangle .
$$

We consider the SU(4) generators $X=S, T, E$. If $|\Psi\rangle$ is a spin, isospin eigenstate $\left|\alpha S M_{S} T M_{T}\right\rangle$, the realizations $\Gamma(S), \Gamma(T)$ of the spin and isospin operators act in the standard way, e.g.

$$
\begin{aligned}
& \Gamma\left(S_{0}\right) \Psi_{\alpha S M_{S} T M_{T}}(\Omega)=M_{S} \Psi_{\alpha S M_{S} T M_{T}}(\Omega) \\
& \Gamma\left(S_{ \pm}\right) \Psi_{\alpha S M_{S} T M_{T}}(\Omega)=\sqrt{\left(S \mp M_{S}\right)\left(S \pm M_{S}+1\right)} \Psi_{\alpha S\left(M_{S} \pm 1\right) T M_{T}}(\Omega) .
\end{aligned}
$$

We shall also be interested in the intrinsic spin or isospin operators which follow from the left realizations of $S$ or $T$ with $S$ or $T$ components acting on the highest-weight or intrinsic states (to the left), to be denoted by a bar, with

$$
\bar{\Gamma}\left(S_{k}\right) \Psi(\Omega)=\left\langle\phi\left|S_{k} R(\Omega)\right| \Psi\right\rangle .
$$

Now if $|\Psi\rangle$ is a spin, isospin eigenstate

$$
\left\langle\phi\left|S_{k} R(\Omega)\right| \alpha S M_{S} T M_{T}\right\rangle=\sum_{K_{S}}\left\langle\phi\left|S_{k}\right| \alpha S K_{S} T K_{T}\right\rangle D_{K_{S} M_{S}}^{S}\left(\Omega_{S}\right) D_{K_{T} M_{T}}^{T}\left(\Omega_{T}\right)
$$

from which it can be seen that

$$
\begin{aligned}
& \bar{S}_{0} D_{K_{S} M_{S}}^{S}\left(\Omega_{S}\right)=K_{S} D_{K_{S} M_{S}}^{S}\left(\Omega_{S}\right) \\
& \bar{S}_{ \pm} D_{K_{S} M_{S}}^{S}\left(\Omega_{S}\right)=\sqrt{\left(S \pm K_{S}\right)\left(S \mp K_{S}+1\right)} D_{\left(K_{S} \mp 1\right) M_{S}}^{S}\left(\Omega_{S}\right)
\end{aligned}
$$

(specific realizations of both the $S_{k}$ and $\bar{S}_{k}$ in terms of the Euler angles $\alpha_{S}, \beta_{S}, \gamma_{S}$ and their derivative operators are given in the appendix. Following [4], the notation $\bar{\Gamma}\left(S_{k}\right)$ is simplified to $\bar{S}_{k}$ when acting on a $D$-function). To gain the rotor vCS realizations of the generators $E$, note that

$$
\begin{aligned}
\Gamma\left(E_{m_{S} m_{T}}\right) \Psi(\Omega) & =\left\langle\phi\left|R(\Omega) E_{m_{S} m_{T}}\right| \Psi\right\rangle \\
& =\left\langle\phi\left|\left(R(\Omega) E_{m_{S} m_{T}} R^{-1}(\Omega)\right) R(\Omega)\right| \Psi\right\rangle \\
& =\sum_{k_{S} k_{T}}\left\langle\phi\left|E_{k_{S} k_{T}} R(\Omega)\right| \Psi\right\rangle D_{k_{S} m_{S}}^{1}\left(\Omega_{s}\right) D_{k_{T} m_{T}}^{1}\left(\Omega_{T}\right) .
\end{aligned}
$$


Using the properties of the highest-weight state (6) and the specific expressions of the generators (4) we see that

$$
\begin{aligned}
& \langle\phi| E_{00}=\frac{1}{2}\left(\lambda_{1}+2 \lambda_{2}+\lambda_{3}\right)\langle\phi| \\
& \langle\phi| E_{ \pm 10}=-\frac{1}{\sqrt{2}}\langle\phi| S_{ \pm} \quad\langle\phi| E_{0 \pm 1}=-\frac{1}{\sqrt{2}}\langle\phi| T_{ \pm} \\
& \left.\langle\phi| E_{ \pm 1 \pm 1}=\frac{1}{2}\langle\phi|\left(-\lambda_{1} \pm S_{0} \pm T_{0}\right) \quad\langle\phi| E_{ \pm 1 \mp 1}=\frac{1}{2}\langle\phi|\left(\lambda_{3} \mp S_{0} \pm T_{0}\right)\right) .
\end{aligned}
$$

These relations lead to

$$
\begin{aligned}
\Gamma\left(E_{m_{s} m_{T}}\right) \Psi(\Omega) & =\left\{\frac{1}{2}\left(\lambda_{1}+2 \lambda_{2}+\lambda_{3}\right) D_{0 m_{s}}^{1}\left(\Omega_{S}\right) D_{0 m_{T}}^{1}\left(\Omega_{T}\right)\right. \\
& -\frac{1}{\sqrt{2}}\left[D_{1 m_{S}}^{1}\left(\Omega_{S}\right) \bar{\Gamma}\left(S_{+}\right)+D_{-1 m_{s}}^{1}\left(\Omega_{S}\right) \bar{\Gamma}\left(S_{-}\right)\right] D_{0 m_{T}}^{1}\left(\Omega_{T}\right) \\
& -\frac{1}{\sqrt{2}} D_{0 m_{S}}^{1}\left(\Omega_{S}\right)\left[D_{1 m_{T}}^{1}\left(\Omega_{T}\right) \bar{\Gamma}\left(T_{+}\right)+D_{-1 m_{T}}^{1}\left(\Omega_{T}\right) \bar{\Gamma}\left(T_{-}\right)\right] \\
& +\frac{1}{2} D_{1 m_{s}}^{1}\left(\Omega_{S}\right) D_{1 m_{T}}^{1}\left(\Omega_{\tau}\right)\left(-\lambda_{1}+\bar{\Gamma}\left(S_{0}\right)+\bar{\Gamma}\left(T_{0}\right)\right) \\
& +\frac{1}{2} D_{-1 m_{S}}^{1}\left(\Omega_{S}\right) D_{-1 m_{T}}^{1}\left(\Omega_{T}\right)\left(-\lambda_{1}-\bar{\Gamma}\left(S_{0}\right)-\bar{\Gamma}\left(T_{0}\right)\right) \\
& +\frac{1}{2} D_{1 m_{S}}^{1}\left(\Omega_{S}\right) D_{-1 m_{T}}^{1}\left(\Omega_{T}\right)\left(\lambda_{3}-\bar{\Gamma}\left(S_{0}\right)+\bar{\Gamma}\left(T_{0}\right)\right) \\
& \left.+\frac{1}{2} D_{-1 m_{s}}^{1}\left(\Omega_{S}\right) D_{1 m_{T}}^{1}\left(\Omega_{T}\right)\left(\lambda_{3}+\bar{\Gamma}\left(S_{0}\right)-\bar{\Gamma}\left(T_{0}\right)\right)\right\}\langle\phi|R(\Omega)| \Psi\rangle .
\end{aligned}
$$

Finally, using the identity (see appendix)

$$
\left[S^{2}, D_{0 m_{S}}^{1}\left(\Omega_{S}\right)\right]=\sqrt{2}\left(D_{1 m_{s}}^{1}\left(\Omega_{S}\right) \bar{\Gamma}\left(S_{+}\right)+D_{-1 m_{S}}^{1}\left(\Omega_{S}\right) \bar{\Gamma}\left(S_{-}\right)\right)+2 D_{0 m_{S}}^{1}\left(\Omega_{S}\right)
$$

and the similar relation for the isospin operators, we obtain

$$
\begin{aligned}
& \Gamma\left(E_{m_{s} m_{T}}\right)=\left\{\frac{1}{2}\left(\lambda_{1}+2 \lambda_{2}+\lambda_{3}\right)+2\right\} D_{0 m_{S}}^{1}\left(\Omega_{S}\right) D_{0 m_{T}}^{1}\left(\Omega_{T}\right) \\
& -\frac{1}{2}\left\{\left[S^{2}, D_{0_{m S}}^{1}\left(\Omega_{S}\right)\right] D_{0 m_{T}}^{1}\left(\Omega_{T}\right)+D_{0 m_{S}}^{1}\left(\Omega_{S}\right)\left[T^{2}, D_{0 m_{T}}^{1}\left(\Omega_{r}\right)\right]\right\} \\
& +\frac{1}{2} D_{1_{m_{S}}}^{1}\left(\Omega_{S}\right) D_{1_{m_{T}}}^{1}\left(\Omega_{T}\right)\left(-\lambda_{1}+\bar{\Gamma}\left(S_{0}\right)+\bar{\Gamma}\left(T_{0}\right)\right) \\
& +\frac{1}{2} D_{-1 m_{s}}^{1}\left(\Omega_{S}\right) D_{-1 m_{T}}^{1}\left(\Omega_{T}\right)\left(-\lambda_{1}-\bar{\Gamma}\left(S_{0}\right)-\bar{\Gamma}\left(T_{0}\right)\right) \\
& +\frac{1}{2} D_{1 m_{s}}^{1}\left(\Omega_{S}\right) D_{-1 m_{r}}^{1}\left(\Omega_{T}\right)\left(\lambda_{3}-\bar{\Gamma}\left(S_{0}\right)+\bar{\Gamma}\left(T_{0}\right)\right) \\
& +\frac{1}{2} D_{-m_{S}}^{1}\left(\Omega_{S}\right) D_{1 m_{r}}^{1}\left(\Omega_{T}\right)\left(\lambda_{3}+\bar{\Gamma}\left(S_{0}\right)-\bar{\Gamma}\left(T_{0}\right)\right) \text {. }
\end{aligned}
$$

Using the symmetrized (normalized) vCs wavefunctions of equation (10) as basis states, a standard $S$ - and $T$-space rotational measure and a standard definition of a spin, isospin reduced double-barred matrix element

$\left\langle K_{S}^{\prime} K_{T}^{\prime} ; S^{\prime} M_{S}^{\prime} T^{\prime} M_{T}^{\prime}\left|\Gamma\left(E_{m_{S} m_{\tau}}\right)\right| K_{S} K_{T} ; S M_{S} T M_{T}\right\rangle$

$$
=\left\langle S M_{S} 1 m_{S} \mid S^{\prime} M_{S}^{\prime}\right\rangle\left\langle T M_{T} 1 m_{T} \mid T^{\prime \prime}{ }_{T}^{\prime \prime}\right\rangle \frac{\left\langle K_{S}^{\prime} K_{T}^{\prime} ; S^{\prime} T^{\prime}\|\Gamma(E)\| K_{S} K_{T} ; S T\right\rangle}{\left[\left(2 S^{\prime}+1\right)\left(2 T^{\prime}+1\right)\right]^{1 / 2}}
$$

together with the well known triple $D$-function integrals, we obtain (for the general $K_{S} K_{T}$ case) the result

$$
\begin{aligned}
\left\langle K_{S} K_{T} ; S^{\prime} T^{\prime} \|\right. & \left.\Gamma(E) \| K_{S} K_{T} ; S T\right\rangle \\
= & {[(2 S+1)(2 T+1)]^{1 / 2}\left\langle S K_{S} 10 \mid S^{\prime} ; K_{S}\right\rangle\left\langle T K_{T} 10 \mid T^{\prime} K_{T}\right\rangle } \\
& \times\left\{\frac{1}{2}\left(\lambda_{1}+2 \lambda_{2}+\lambda_{3}\right)+2-\frac{1}{2} S^{\prime}\left(S^{\prime}+1\right)+\frac{1}{2} S(S+1)\right. \\
& \left.-\frac{1}{2} T^{\prime}\left(T^{\prime}+1\right)+\frac{1}{2} T(T+1)\right\} \\
\left\langle( K _ { S } \pm 1 ) \left( K_{Y}\right.\right. & \left. \pm 1) ; S^{\prime} T^{\prime}\|\Gamma(E)\| K_{S} K_{T} ; S T\right\rangle \\
= & \frac{1}{2}[(2 S+1)(2 T+1)]^{1 / 2}\left\langle S K_{S} 1 \pm 1 \mid S^{\prime}\left(K_{S} \pm 1\right)\right\rangle \\
& \times\left\langle T K_{T} 1 \pm 1 \mid T^{\prime}\left(K_{T} \pm 1\right)\right\rangle\left(-\lambda_{1} \pm K_{S} \pm K_{T}\right)
\end{aligned}
$$




$$
\begin{aligned}
\left\langle\left(K_{S} \pm 1\right)\left(K_{T} \mp 1\right) ; S^{\prime} T^{\prime}\|\Gamma(E)\| K_{S} K_{T} ; S T\right\rangle & \\
= & \frac{1}{2}[(2 S+1)(2 T+1)]^{1 / 2}\left\langle S K_{S} 1 \pm 1 \mid S^{\prime}\left(K_{S} \pm 1\right)\right\rangle \\
& \times\left\{T K_{T} 1 \mp 1\left|T^{\prime}\left(K_{T} \mp 1\right)\right\rangle\left(\lambda_{3} \mp K_{S} \pm K_{T}\right) .\right.
\end{aligned}
$$

Note that states with $K_{S}+K_{T}=0$ or 1 require special treatment due to the symmetrized form of the basis functions of equation (10). Thus off-diagonal matrix elements in $K_{S} K_{T}$ with $K_{S} K_{T}$ or $K_{S}^{\prime} K_{T}^{\prime}=00$ require an additional $\sqrt{2}$, e.g.

$\left\langle 11 ; S^{\prime} T^{\prime}\|\Gamma(E)\| 00 ; S T\right\rangle$

$$
=\sqrt{2} \times \frac{1}{2}[(2 S+1)(2 T+1)]^{1 / 2}\left\langle S 011 \mid S^{\prime} 1\right\rangle\left\langle T 011 \mid T^{\prime} 1\right\rangle\left(-\lambda_{1}\right)
$$

$\left\langle 00 ; S^{\prime} T^{\prime}\|\Gamma(E)\| 11 ; S T\right\rangle$

$$
=\sqrt{2} \times \frac{1}{2}[(2 S+1)(2 T+1)]^{1 / 2}\left\langle S 11-1 \mid S^{\prime} 0\right\rangle\left\langle T 11-1 \mid T^{\prime} 0\right\rangle\left(-\lambda_{1}-2\right)
$$

with similar factors of $\sqrt{2}$ needed for $K_{S} K_{T}=00, K_{S}^{\prime} K_{T}^{\prime}=1-1$; or $K_{S} K_{T}=1-1$, $K_{S}^{\prime} K_{T}^{\prime}=00$.

In addition,

$\left\langle\frac{1}{2} \frac{1}{2} ; S^{\prime} T^{\prime}\|\Gamma(E)\| \frac{1}{2} \frac{1}{2} ; S T\right\rangle$

$$
\begin{aligned}
= & {[(2 S+1)(2 T+1)]^{1 / 2}\left\{\left[\frac{1}{2}\left(\lambda_{1}+2 \lambda_{2}+\lambda_{3}\right)+2-\frac{1}{2} S^{\prime}\left(S^{\prime}+1\right)+\frac{1}{2} S(S+1)\right.\right.} \\
& \left.-\frac{1}{2} T^{\prime}\left(T^{\prime}+1\right)+\frac{1}{2} T(T+1)\right]\left\langle S \frac{1}{2} 10 \mid S^{\prime} \frac{1}{2}\right\rangle\left\langle T \frac{1}{2} 10 \mid T^{\prime} \frac{1}{2}\right\rangle \\
& \left.+\frac{1}{2}(-1)^{\lambda_{2}+\lambda_{3}+S+T}\left(-\lambda_{1}-1\right)\left\langle S-\frac{1}{2} 11 \mid S^{\prime} \frac{1}{2}\right\rangle\left\langle T-\frac{1}{2} 11 \mid T^{\prime} \frac{1}{2}\right\rangle\right\}
\end{aligned}
$$

$\left\langle\frac{1}{2}-\frac{1}{2} ; S T\|\Gamma(E)\| \frac{1}{2}-\frac{1}{2} ; S T\right\rangle$

$$
\begin{aligned}
= & {[(2 S+1)(2 T+1)]^{1 / 2}\left\{\left[\frac{1}{2}\left(\lambda_{1}+2 \lambda_{2}+\lambda_{3}\right)+2-\frac{1}{2} S^{\prime}\left(S^{\prime}+1\right)+\frac{1}{2} S(S+1)\right.\right.} \\
& \left.\left.-\frac{1}{2} T^{\prime}\left(T^{\prime}+1\right)\right)+\frac{1}{2} T(T+1)\right]\left\langle S \frac{1}{2} 10 \mid S^{\prime} \frac{1}{2}\right\rangle\left\langle T-\frac{1}{2} 10 \mid T^{\prime}-\frac{1}{2}\right\rangle \\
& \left.\left.+\frac{1}{2}(-1)^{\lambda_{2}+\lambda_{3}+S+T}\left(\lambda_{3}+1\right)\left\langle S-\frac{1}{2} 11 \mid S^{\prime} \frac{1}{2}\right\rangle\left\langle T \frac{1}{2} 1-1\right| T^{\prime}-\frac{1}{2}\right\}\right\} .
\end{aligned}
$$

Also,

$$
\begin{aligned}
& \left\langle 01 ; S^{\prime} T^{\prime}\|\Gamma(E)\| 10 ; S T\right\rangle=[(2 S+1)(2 T+1)]^{1 / 2} \\
& \quad \times \frac{1}{2}\left(\lambda_{3}+1\right)\left\langle S 11-1 \mid S^{\prime} 0\right\rangle\left\langle T 01+1 \mid T^{\prime} 1\right\rangle\left(1+(-1)^{\lambda_{2}+\lambda_{3}+S^{\prime}+T}\right) \\
& \left\langle 10 ; S^{\prime} T^{\prime}\|\Gamma(E)\| 01 ; S T\right\rangle=[(2 S+1)(2 T+1)]^{1 / 2} \\
& \times \frac{1}{2}\left(\lambda_{3}+1\right)\left\langle S 011 \mid S^{\prime} 1\right\rangle\left\langle T 11-1 \mid T^{\prime} 0\right\rangle\left(1+(-1)^{\lambda_{2}+\lambda_{3}+S+T^{\prime}}\right) .
\end{aligned}
$$

All other cases are given by the generic formulae of equations (22).

There remains the task of establishing the symmetrized form of the double rotor basis functions of equation (10). This will be achieved by constructing the generic SU(4) irreducible representation $\left[f_{1} f_{2} f_{3} f_{4}\right]=\left[\lambda_{1}+\lambda_{2}+\lambda_{3}, \lambda_{2}+\lambda_{3}, \lambda_{3}\right.$ 0] by a symmetric coupling of the states of the irreps $\left[\lambda_{1} 000\right],\left[\lambda_{2} \lambda_{2} 00\right]$, and $\left[\lambda_{3} \lambda_{3} \lambda_{3} 0\right]$; starting with the basic states [1000] $S T=\frac{1}{2} \frac{1}{2},[1110] S T=\frac{1}{2} \frac{1}{2}$; and [1100] $S T=10$ or 01 . The build-up process requires the $D$-function build-up formula

$D_{K_{1} M_{1}}^{J_{1}}(\Omega) D_{K_{2} M_{2}}^{J_{2}}(\Omega)=\sum_{J} D_{K M}^{J}(\Omega)\left\langle J_{1} K_{1} J_{2} K_{2} \mid J K\right\rangle\left\langle J_{1} M_{1} J_{2} M_{2} \mid J M\right\rangle$.

The starting states [1000] and [1110], the irreps of the single-particle creation and annihilation operators, are given by

$\Psi_{(1 / 2) M_{s},(1 / 2) M_{\top}}^{[1000]}(\Omega)$

$$
=\frac{1}{\sqrt{2}} \frac{2}{8 \pi^{2}}\left\{D_{+(1 / 2) M_{S}}^{1 / 2}\left(\Omega_{S}\right) D_{+(1 / 2) M_{T}}^{1 / 2}\left(\Omega_{T}\right)-D_{-(1 / 2) M_{S}}^{1 / 2}\left(\Omega_{S}\right) D_{-(1 / 2) M_{T}}^{1 / 2}\left(\Omega_{T}\right)\right\}
$$


whereas

$\Psi_{(1 / 2) M_{s},-(1 / 2) M_{T}(\Omega)}^{[110]}$

$$
=\frac{1}{\sqrt{2}} \frac{2}{8 \pi^{2}}\left\{D_{+(1 / 2) M_{S}}^{1 / 2}\left(\Omega_{S}\right) D_{-(1 / 2) M_{T}}^{1 / 2}\left(\Omega_{T}\right)+D_{-(1 / 2) M_{S}}^{1 / 2}\left(\Omega_{S}\right) D_{+(1 / 2) M_{T}}^{1 / 2}\left(\Omega_{T}\right)\right\} .
$$

In general, totally symmetric double rotor coherent wavefunctions of the type of equations (8) and (10) can have $S T$ values (with $T=S$ ) up to arbitrarily high $S$. The phase of equation (29) is chosen to ensure that the $S T$ values terminate at $S T=S S$, with $S^{\prime}=\frac{1}{2} \lambda_{1}=\frac{1}{2}$. Totally symmetric states with $\lambda_{1}>1\left(\lambda_{2}=\lambda_{3}=0\right)$ can be built by successive action with $\Gamma(E)$. This leads to the (normalized) state

$$
\Psi_{S M_{S}, T=S M_{T}}^{\left(\lambda_{1}, 00\right)}(\Omega)=\sum_{K=-S}^{+S} \frac{(-1)^{\left(K-\frac{1}{2} \lambda_{1}\right)}}{[2 S+1]^{1 / 2}} \frac{2 S+1}{8 \pi^{2}} D_{K M_{S}}^{S}\left(\Omega_{S}\right) D_{K M_{T}}^{S}\left(\Omega_{T}\right)
$$

(the factor $\frac{1}{2} \lambda_{1}$ in the phase is arbitrary; it is chosen merely for convenience to make the normalization factor real).

Similarly, the normalized state for the irrep $\left[\lambda_{3} \lambda_{3} \lambda_{3} 0\right]$ conjugate to the totally symmetric irrep, again with $T=S$, is given by

$\Psi_{S M_{s}, T=S M_{T}}^{\left[\lambda_{3} \lambda_{3} \lambda_{3} 0\right]}(\Omega)=\sum_{K=-s}^{+S} \frac{1}{[2 S+1]^{1 / 2}} \frac{2 S+1}{8 \pi^{2}} D_{K M_{s}}^{S}\left(\Omega_{S}\right) D_{-K M_{r}}^{S}\left(\Omega_{T}\right)$.

Note the different relative phases. Together with (22) these lead to the reduced matrix elements

$$
\begin{aligned}
\langle[y 000] S+1 S+1\|\Gamma(E)\|[y 000] S S\rangle & =\langle[y y y 0] S+1 S+1\|\Gamma(E)\|[y y y 0] S S\rangle \\
& =[(2 S+1)(2 S+3)]^{1 / 2}\left(\frac{1}{2} y-S\right) .
\end{aligned}
$$

Note the termination at a maximum possible $S$ value, $S_{\max }=\frac{1}{2} y$. Similarly,

$$
\begin{aligned}
\langle[y 000] S S\|\Gamma(E)\|[y 000] S+1 S+1\rangle & =\langle[y y y 0] S S\|\Gamma(E)\|[y y y 0] S+1 S+1\rangle \\
& =[(2 S+1)(2 S+3)]^{1 / 2}\left(\frac{1}{2} y+S+2\right) .
\end{aligned}
$$

Finally, the irreps $\left[\lambda_{2} \lambda_{2} 00\right]$ have $K_{S}=K_{T}=0$ only (see equation (9)). The $S T$ values are restricted by the requirement $\lambda_{2}+S+T=$ even, starting with $S T=00$ for $\lambda_{2}=$ even and with $S T=10,01$ for $\lambda_{2}=$ odd.

The symmetric coupling of irreps $\left[\lambda_{1} 000\right]$ and $\left[\lambda_{2} \lambda_{2} 00\right]$ to resultant $\left[\left(\lambda_{1}+\lambda_{2}\right) \lambda_{2} 00\right]$ involves products of double rotor functions of the type

$$
\left\{D_{K_{1} M_{S_{1}}}^{S_{1}}\left(\Omega_{S}\right) D_{K_{1} M_{T_{1}}}^{S_{1}}\left(\Omega_{T}\right)+(-1)^{2 S_{1}} D_{-K_{1} M_{S_{1}}}^{S_{1}}\left(\Omega_{S}\right) D_{-K_{1} M_{T_{1}}}^{S_{1}}\left(\Omega_{T}\right)\right\} D_{0 M_{S_{2}}}^{S_{2}}\left(\Omega_{S}\right) D_{0 M_{T_{2}}}^{T_{2}}\left(\Omega_{T}\right) .
$$

The build-up formula (28) and the symmetry of the Wigner coefficients leads to final double rotor functions of type

$$
\left\{D_{K_{\mathrm{t}} M_{S}}^{S}\left(\Omega_{S}\right) D_{K_{\mathrm{t}} M_{T}}^{T}\left(\Omega_{T}\right)+(-1)^{S+T-S_{2}-T_{2}} D_{-K_{1} M_{S}}^{S}\left(\Omega_{S}\right) D_{-K_{1} M_{T}}^{T}\left(\Omega_{T}\right)\right\}
$$

where $\left(S_{2}+T_{2}\right)$ in the phase factor can be replaced by $\pm \lambda_{2}$ due to the requirement $\lambda_{2}+S_{2}+T_{2}=$ even. The further symmetric coupling of irreps $\left[\left(\lambda_{1}+\lambda_{2}\right) \lambda_{2} 00\right]$ with $\left[\lambda_{3} \lambda_{3} \lambda_{3} 0\right]$ to resultant $\left[\left(\lambda_{1}+\lambda_{2}+\lambda_{3}\right)\left(\lambda_{2}+\lambda_{3}\right) \lambda_{3} 0\right]$ leads to products of the double rotor functions of type (35b) with functions of type

$$
\left\{D_{K_{3} M_{S_{3}}}^{S_{3}}\left(\Omega_{S}\right) D_{-K_{3} M_{T_{3}}}^{T_{3}=S_{3}}\left(\Omega_{T}\right)+D_{-K_{3} M_{S_{3}}}^{S_{3}}\left(\Omega_{S}\right) D_{K_{3} M_{\tau_{3}}}^{S_{3}}\left(\Omega_{T}\right)\right\} .
$$


Application of equation (28) and of the symmetry properties of the Wigner coefficients lead to final double rotor functions of type

$\left\{D_{K_{S}^{\prime} M_{S}^{\prime}}^{S^{\prime}}\left(\Omega_{S}\right) D_{K_{J}^{\prime} M_{T}^{\prime}}^{r^{\prime}}\left(\Omega_{T}\right)+(-1)^{\lambda_{2}+S^{\prime}+T^{\prime}-2 S_{3}} D_{-K_{S}^{\prime} M_{S}^{\prime}}^{S^{\prime}}\left(\Omega_{S}\right) D_{-K_{T}^{\prime} M_{T}^{\prime}}^{T^{\prime}}\left(\Omega_{T}\right)\right\}$

with $K_{S}^{\prime}, K_{T}^{\prime}=\left(K_{1}+K_{3}\right),\left(K_{1}-K_{3}\right)$ or $\left(K_{1}-K_{3}\right),\left(K_{1}+K_{3}\right)$. Since $2 S_{3}=$ even (or odd) for $\lambda_{3}=$ even (or odd), the basic phase of the symmetrized double rotor functions of equation (10) has been established.

\section{The $\mathcal{K}$ matrices for the double rotor $S U(4) \supset S U(2) \times S U(2)$ states}

The double rotor vCS representations of the SU(4) generators, the $\Gamma(X)$ of section 2 , are not unitary. This can be seen at once by noting that the reduced matrix elements of $\Gamma(E)$ change by more than the phase factor $(-1)^{S+T-S^{\prime \prime}-T^{\prime}}$ under bra-ket interchange. (Compare, for example, equations (33) and (34).) In vCS theory, the $\Gamma(X)$ are converted to unitary form $\gamma(X)$ via the similarity transformation

$$
\gamma(X)=\mathcal{K}^{-1} \Gamma(X) \mathcal{K} .
$$

If $X$ is a double spherical tensor operator of type $X_{m_{S} m_{5}}^{S T}$, the adjoint operators in the unitary representation must satisfy [4]

$$
\gamma^{\dagger}\left(X_{m_{S} m_{T}}\right)=(-1)^{m_{S}+m_{T}} \gamma\left(X_{-m_{s},-m_{T}}\right) \text {. }
$$

Substitution into (36) leads to

$$
\mathcal{K} \mathcal{K}^{\dagger}(-1)^{m_{S}+m_{T}} \Gamma^{\dagger}\left(X_{-m_{S_{1}-m_{T}}}\right)=\Gamma\left(X_{m_{S} m_{T}}\right) \mathcal{K} \mathcal{K}^{\dagger} \text {. }
$$

Following Rowe et al [4], we define

$$
\Gamma^{*}\left(X_{m_{S} m_{T}}\right)=(-1)^{m_{S}+m_{T}} \Gamma^{\dagger}\left(X_{-m_{s}-m_{T}}\right)
$$

with reduced matrix elements

$\left\langle K_{S}^{\prime} K_{T}^{\prime} ; S^{\prime} T^{\prime}\left\|\Gamma^{\#}\right\| K_{S} K_{T} ; S T\right\rangle=(-1)^{S+T-S^{\prime}-T^{\prime}}\left\langle K_{S} K_{T} ; S T\|\Gamma\| K_{S}^{\prime} K_{T}^{\prime} ; S^{\prime} T^{\prime}\right\rangle$.

As usual, $\mathcal{K} \mathcal{K}^{\dagger}$ commutes with the subgroup generators $S$ and $T$ so that $\mathcal{K} \mathcal{K}^{\dagger}$ is block diagonal in $S$ and $T$ and is one-dimensional for the minimum $S, T$ values of $00, \frac{1}{2} \frac{1}{2}$, or 10,01 . As always, we choose the starting value of $\mathcal{K}$ to be +1 for states with these minimum $S, T$ values, the starting points for the vCS $\mathcal{K}$-matrix constructions. $\mathcal{K K}^{\dagger}$ matrices for larger values of $S, T$ can then be calculated by the usual recursion relations. For example, equations (38) and (40) lead to the recursion relation

$$
\begin{array}{r}
\sum_{K_{S_{2}}^{\prime} K_{T_{2}}^{\prime}}\left(\mathcal{K} \mathcal{K}^{\dagger}\left(S^{\prime}, T^{\prime}\right)\right)_{K_{S_{1}}^{\prime} K_{T_{1}}^{\prime} ; K_{S_{2}}^{\prime} K_{T_{2}}^{\prime}}\left\langle K_{S_{2}} K_{T_{2}} ; S T\|\Gamma(E)\| K_{S_{2}}^{\prime} K_{T_{2}}^{\prime} ; S^{\prime} T^{\prime}\right\rangle(-1)^{S+T-S^{\prime}-T^{\prime}} \\
=\sum_{K_{S_{1}} K_{T_{1}}}\left\langle K_{S_{1}}^{\prime} K_{T_{1}}^{\prime} ; S^{\prime} T^{\prime}\|\Gamma(E)\| K_{S_{1}} K_{T_{1}} ; S T\right\rangle\left(\mathcal{K} \mathcal{K}^{\dagger}(S, T)\right)_{K_{S_{1}} K_{T_{1}} ; K_{S_{2}} K_{T_{2}}} .
\end{array}
$$

Different choices of the fixed $K_{S_{1}}^{\prime} K_{T_{1}}^{\prime}$ and $K_{S_{2}} K_{T_{2}}$ are usually sufficient to determine the $\mathcal{K} \mathcal{K}^{\dagger}\left(S^{\prime}, T^{\prime}\right)$ matrices from the known $\mathcal{K} \mathcal{K}^{\dagger}(S, T)$ values. In some (rare) cases a second recursion formula may be needed. Following Rowe et al [4], we use

$$
\begin{aligned}
& \sum_{K_{S_{2}}^{\prime} K_{T_{2}}^{\prime}} \sum_{K_{S_{3}}^{\prime} K_{T_{3}}^{\prime}}\left(\mathcal{K} \mathcal{K}^{\dagger}\left(S^{\prime}, T^{\prime}\right)\right)_{K_{S_{1}}^{\prime} K_{T_{1}}^{\prime} ; K_{S_{2}}^{\prime} K_{T_{2}}^{\prime}\left\langle K_{S_{2}}^{\prime} K_{T_{2}}^{\prime} ; S^{\prime} T^{\prime}\left\|\Gamma^{\#}(E)\right\| K_{S_{3}}^{\prime} K_{T_{3}}^{\prime} ; S T\right\rangle} \\
& \quad \times\left\langle K_{S_{3}}^{\prime} K_{T_{3}}^{\prime} ; S^{\prime} T^{\prime}\left\|\Gamma^{\#}(E)\right\| K_{S_{2}} K_{T_{2}} ; S T\right\rangle \\
&=\sum_{K_{S_{1}} K_{T_{1}}} \sum_{K_{S_{3}}^{\prime} K_{T_{3}}^{\prime}}\left\langle K_{S_{1}}^{\prime} K_{T_{1}}^{\prime} ; S^{\prime} T^{\prime}\|\Gamma(E)\| K_{S_{3}}^{\prime} K_{T_{3}}^{\prime} ; S^{\prime} T^{\prime}\right\rangle \\
& \times\left\langle K_{S_{3}}^{\prime} K_{T_{3}}^{\prime} ; S^{\prime} T^{\prime}\|\Gamma(E)\| K_{S_{1}} K_{T_{1}} ; S T\right\rangle\left(\mathcal{K} \mathcal{K}^{\dagger}(S, T)\right)_{K_{S_{1}} K_{T_{1}} ; K_{S_{2}} K_{T_{2}}} .
\end{aligned}
$$


This follows from the matrix element of relation (38):

$$
\begin{aligned}
\left\langle K_{S_{1}}^{\prime} K_{T_{1}}^{\prime} ; S^{\prime} T^{\prime}\left\|\mathcal{K} \mathcal{K}^{\dagger} \Gamma^{\#}(E)\right\| K_{S_{3}}^{\prime} K_{T_{3}}^{\prime} ; S^{\prime} T^{\prime}\right\rangle \\
=\left\{K_{S_{1}}^{\prime} K_{T_{1}}^{\prime} ; S^{\prime} T^{\prime}\left\|\Gamma(E) \mathcal{K} \mathcal{K}^{\dagger}\right\| K_{S_{3}}^{\prime} K_{T_{3}}^{\prime} ; S^{\prime} T^{\prime}\right\} .
\end{aligned}
$$

This is then multiplied by $\left\langle K_{S_{3}}^{\prime} K_{T_{3}}^{\prime} ; S^{\prime} T^{\prime}\left\|\Gamma^{\#}(E)\right\| K_{S_{2}} K_{T_{2}} ; S T\right\rangle$ and summed over all $K_{S_{3}}^{\prime} K_{T_{3}}^{\prime}$. The right-hand side of this relation then leads to

$$
\begin{aligned}
& \sum_{K_{S_{3}}^{\prime} K_{T_{3}}^{\prime}}\left\langle K_{S_{1}}^{\prime} K_{T_{1}}^{\prime} ; S^{\prime} T^{\prime}\left\|\Gamma(E) \mathcal{K} \mathcal{K}^{\dagger}\right\| K_{S_{3}}^{\prime} K_{T_{3}}^{\prime} ; S^{\prime} T^{\prime}\right\rangle\left\langle K_{S_{3}}^{\prime} K_{T_{3}}^{\prime} ; S^{\prime} T^{\prime}\left\|\Gamma^{\#}(E)\right\| K_{S_{2}} K_{T_{2}} ; S T\right\rangle \\
&=\sum_{K_{S_{3}}^{\prime} K_{T_{3}}^{\prime}}\left\langle K_{S_{1}}^{\prime} K_{T_{1}}^{\prime} ; S^{\prime} T^{\prime}\|\Gamma(E)\| K_{S_{3}}^{\prime} K_{T_{3}}^{\prime} ; S^{\prime} T^{\prime}\right\rangle \\
& \times\left\langle K_{S_{3}}^{\prime} K_{T_{3}}^{\prime} ; S^{\prime} T^{\prime}\left\|\mathcal{K} \mathcal{K}^{\dagger} \Gamma^{\sharp}(E)\right\| K_{S_{2}} K_{T_{2}} ; S T\right\rangle \\
&= \sum_{K_{S_{3}}^{\prime} K_{T_{3}}^{\prime}}\left\langle K_{S_{1}}^{\prime} K_{T_{1}}^{\prime} ; S^{\prime} T^{\prime}\|\Gamma(E)\| K_{S_{3}}^{\prime} K_{T_{3}}^{\prime} ; S^{\prime} T^{\prime}\right\rangle \\
& \times\left\langle K_{S_{3}}^{\prime} K_{T_{3}}^{\prime} ; S^{\prime} T^{\prime}\left\|\Gamma(E) \mathcal{K} \mathcal{K}^{\dagger}\right\| K_{S_{2}} K_{T_{2}} ; S T\right\rangle .
\end{aligned}
$$

In the first step of this process, since $\mathcal{K} \mathcal{K}^{\dagger}$ is diagonal in the $S, T$ quantum numbers, it can be pushed through to the right since the sum over $K_{S_{3}}^{\prime} K_{T_{3}}^{\prime}$ effectively yields a sum over a complete set of intermediate states. (I am indebted to D J Rowe for enlightenment on this point.) The second step uses equation (38).

Using relations (41) and (42) the $\mathcal{K} \mathcal{K}^{\dagger}(S, T)$ matrices can be evaluated, starting with the minimum $S, T$ values for a particular irreducible representation of SU(4). To make the transformation from the vCS basis $\left|K_{S} K_{T} ; S M_{S} T M_{T}\right\rangle$ to the Hermitian basis, it is sufficient to diagonalize the Hermitian $K K^{\dagger}$ matrices via a unitary matrix, $U$ :

$$
\mathcal{K} \mathcal{K}^{\dagger} U^{\dagger}=\lambda=\lambda_{i} \delta_{i j}
$$

with $i, j=1, \ldots$, the dimension of $\mathcal{K} \mathcal{K}^{\dagger}(S, T)$, yielding

$$
\mathcal{K}_{K_{S} K_{T}, i}=(U)_{K_{S} K_{T}, i}^{\dagger} \sqrt{\lambda_{i}} \quad\left(\mathcal{K}^{-1}\right)_{i, K_{S} K_{T}}=\frac{1}{\sqrt{\lambda_{i}}} U_{i, K_{S} K_{T}}
$$

The map to the unitary realization of the operators, $X$, via equation (36), can then be mapped back to ordinary Hilbert space to yield, e.g.

$$
\left\langle i^{\prime} ; S^{\prime} T^{\prime}\|E\| i, S T\right\rangle=\sum_{K_{S} K_{T}} \sum_{K_{S}^{\prime} K_{T}^{\prime}}\left(\mathcal{K}^{-1}\right)_{i^{\prime}, K_{S}^{\prime} K_{T}^{\prime}}\left\langle K_{S}^{\prime} K_{T}^{\prime} ; S^{\prime} T^{\prime}\|\Gamma(E)\| K_{S} K_{T}: S T\right\rangle(\mathcal{K})_{K_{S} K_{T}, i}
$$

Since the reduced matrix elements of $\Gamma(E)$ are given simply in terms of ordinary angular momentum Wigner coefficients (equations (22)-(27)), a very simple form of the matrix elements of $E$ has been achieved for ireducible representations for which simple expressions can be given for the $\mathcal{K}$ matrices. For this purpose analytic expressions will be given for the $\mathcal{K} \mathcal{K}^{\dagger}$ matrix elements for many of the simpler SU(4) irreducible representations starting with $[f]=\left[\lambda_{2} \lambda_{2} 00\right]$ and the irreps which can be built from a coupling of $\left[\lambda_{2} \lambda_{2} 00\right]$ with $\left[\lambda_{1} 000\right]$, with $\lambda_{1}=1,2,3, \ldots, a$, leading to $\mathcal{K} \mathcal{K}^{\dagger}$ matrices of dimension $\leqslant a \times a$. For the irrep [yy00] with $\lambda_{1}=\lambda_{3}=0$ and hence $K_{S} K_{T}=00$ only, the $\mathcal{K} \mathcal{K}^{\dagger}$ matrix is one-dimensional and follows from the starting value of unity for $S T=00$ ( $y=$ even), or 
$S T=10(y=$ odd). A straightforward application of the recursion relation (41) gives for [yy00], with $K_{S} K_{T}=00$ only and $y+S+T=$ even only:

$$
\begin{aligned}
& \mathcal{K K}^{\dagger}(S, T)=\frac{\text { Num }}{(y-S-T) ! !(y+S+T+2) ! !(y+1+S-T) ! !(y+1-S+T) ! !} \\
& \text { Num }=y ! !(y+2) ! !(y+1) ! !(y+1) ! ! \quad \text { for } y=\text { even } \\
& =(y-1) ! !(y+3) ! !(y+2) ! ! y ! ! \quad \text { for } y=\text { odd }
\end{aligned}
$$

where the double factorial is defined by $y ! !=y(y-2) \cdots 2$ (or 1) for $y=$ even(odd). (The numerators assure that $0 ! ! \equiv 1$.) The next case (with $a=1$ ) leads to the irreducible representations $[y y-100],\left\{\lambda_{1}=1, \lambda_{2}=y-1, \lambda_{3}=0\right\}$, and its conjugate [yy 1$],\left\{\lambda_{1}=0, \lambda_{2}=y-1, \lambda_{3}=1\right\}$, again with one-dimensional $\mathcal{K} \mathcal{K}^{\dagger}$ matrices, with $K_{S} K_{T}=\frac{1}{2} \frac{1}{2}$ and $\frac{1}{2}-\frac{1}{2}$, respectively. In this case we must distinguish between states with $y+S+T=$ even or odd. For $[y y-100]$, with $K_{S} K_{T}=\frac{1}{2} \frac{1}{2}$, or [yy10], with $K_{S} K_{T}=\frac{1}{2}-\frac{1}{2}$; For $y+S+T=$ even:

$\mathcal{K} \mathcal{K}^{\dagger}(S, T)=\frac{\text { Num }}{(y-S-T) ! !(y+S+T+2) ! !(y+S-T) ! !(y-S+T) ! !}$.

For $y+S+T=$ odd:

$$
\mathcal{K K}^{\dagger}(S, T)=\frac{\text { Num }}{(y-1-S-T) ! !(y+1+S+T) ! !(y+1+S-T) ! !(y+1-S+T) ! !}
$$

$$
\begin{aligned}
\text { Num } & =(y-2) ! !(y+2) ! !(y+1) ! !(y+1) ! ! & & \text { for } y=\text { even } \\
& =(y-1) ! !(y+3) ! ! y ! ! y ! ! & & \text { for } y=\text { odd }
\end{aligned}
$$

As a specific example consider the matrix element of $E$ for the case $y+S+T=$ even, $S^{\prime} T^{\prime}=S+1 T+1$. Equations (24) and (47a) give

$$
\begin{aligned}
& \langle[y y-100] S+1 T+1\|E\|[y y-100] S T\rangle \\
& =\frac{\mathcal{K}(S, T)}{\mathcal{K}(S+1, T+1)} \frac{(y-S-T)}{4}\left[\frac{(2 S+1)(2 S+3)(2 T+1)(2 T+3)}{(S+1)(T+1)}\right]^{1 / 2} \\
& =\left[\frac{(y+S+T+4)(y-S-T)(2 S+1)(2 S+3)(2 T+1)(2 T+3)}{16(S+1)(T+1)}\right]^{1 / 2} \text {. }
\end{aligned}
$$

Equations (25) and (47a) give the same result for the reduced matrix element of $E$ for the irrep [yy10]. Except for a somewhat different phase convention, this and similar results for all irreducible representations with one-dimensional $\mathcal{K} \mathcal{K}^{\dagger}$ matrices agree with the analytic expressions of [9]. The spin-isospin reduced matrix element of $E$ is related to the $S U(4) \supset S U(2) \times S U(2)$ reduced Wigner coefficient with outer multiplicity label [9] $\rho=1$, via

$$
\left\langle[f] S T ;[211] 11 \|[f] S^{\prime} T^{\prime}\right\rangle_{\rho=1}=\frac{\left.\langle[f]] S^{\prime} T^{\prime}\|E\|[f] S T\right\rangle}{\left[\left(2 S^{\prime}+1\right)\left(2 T^{\prime}+1\right) C_{S U_{4}}\right]^{1 / 2}}
$$

where $C_{\mathrm{SU}_{4}}$ is the quadratic Casimir invariant, $C_{\mathrm{SU}_{4}}=P(P+4)+P^{\prime}\left(P^{\prime}+2\right)+P^{\prime 2}$.

The next case (with $a=2$ ) includes the irreps $[y+2, y 00]$, its conjugate $[y+2, y+2,20]$ and $[y+1, y 10]$.

For $[y+2, y 00]$, with $K_{S} K_{T}=11$ or 00 , the $\mathcal{K} \mathcal{K}^{\dagger}$ matrices are one-dimensional for $y+S+T=$ odd (since $K_{S} K_{T}=00$ does not exist for this case); leading to: 
For $[y+2, y 00], y+S+T=$ odd:

$\left(\mathcal{K K}^{\dagger}(S, T)\right)_{1,11}$

$$
=\frac{\text { Num }}{(2(y+3+S+T) ! !(y+1-S-T) ! !(y+2+S-T) ! ! \times(y+2-S+T) ! !}
$$

with

$$
\begin{aligned}
\text { Num } & =(y+4) ! ! y ! !(y+3) ! !(y+1) ! ! & & \text { for } y=\text { even } \\
& =(y+5) ! !(y-1) ! !(y+2) ! !(y+2) ! ! & & \text { for } y=\text { odd. }
\end{aligned}
$$

For $[y+2, y 00], y+S+T=$ even, the $2 \times 2 \mathcal{K} \mathcal{K}^{\dagger}$ matrices are given by

$$
\begin{aligned}
& \left(\mathcal{K} \mathcal{K}^{\dagger}(S, T)\right)_{11,11}=\frac{1}{2}[(y+3)(y+4)-S(S+1)-T(T+1)] C F \\
& \left(\mathcal{K} \mathcal{K}^{\dagger}(S, T)\right)_{00,00}=[(y+3)(y+2)-S(S+1)-T(T+1)] C F \\
& \left(\mathcal{K} \mathcal{K}^{\dagger}(S, T)\right)_{11,00}=-[2 S(S+1) T(T+1)]^{1 / 2} \mathrm{CF}
\end{aligned}
$$

with the common factor given by

$$
\mathrm{CF}=\frac{\text { Num }}{(y+4+S+T) ! !(y+2-S-T) ! !(y+3+S-T) ! !(y+3-S+T) ! !}
$$

with Num given by (50b). Equations (50) and (51) also give the $\mathcal{K} \mathcal{K}^{\dagger}$ matrices for the irrep $[y+2, y+2,20]$ if the $K_{S} K_{T}$ values 11,00 are replaced by $1-1,00$. The Hermitian matrix $\mathcal{K K}^{\dagger}$ is real and hence symmetric.

We also note that the $\mathcal{K} \mathcal{K}^{\dagger}$ matrix for the bounding value, $S+T=y+2$, with the maximum possible $S+T$ value, is singular. In this case the $\mathcal{K} \mathcal{K}^{\dagger}$ matrix

$$
\left(\begin{array}{cc}
(y+3-S)(S+1) \mathrm{CF} & -[2 S(S+1)(y+2-S)(y+3-S)]^{1 / 2} \mathrm{CF} \\
-[2 S(S+1)(y+2-S)(y+3-S)]^{1 / 2} \mathrm{CF} & 2 S(y+2-S) \mathrm{CF}
\end{array}\right)
$$

clearly has one zero eigenvalue (with a redundant state). The physical subspace with eigenvalue $\lambda_{1}=\left\{\left[(y+3)+3 S(y+2)-3 S^{2}\right] C F\right\}$ leads to the single physical state, $i=1$, with

$$
\begin{aligned}
& \left(\mathcal{K}^{-1}\right)_{i=1 ; K_{S} K_{T}=11}=\frac{1}{\lambda_{1}}[(y+3-S)(S+1) \mathrm{CF}]^{1 / 2}=\frac{1}{\lambda_{1}}(\mathcal{K})_{K_{S} K_{T}=11 ; i=1} \\
& \left(\mathcal{K}^{-1}\right)_{i=1 ; K_{S} K_{T}=00}=\frac{1}{\lambda_{1}}[2 S(y+2-S) \mathrm{CF}]^{1 / 2}=\frac{1}{\lambda_{1}}(\mathcal{K})_{K_{S} K_{T}=0 ; i=1} .
\end{aligned}
$$

Finally, for $[y+1 y 10]$, with $y+S+T=$ even:

$\left(\mathcal{K} \mathcal{K}^{\dagger}(S, T)\right)_{10,10}=\left(\mathcal{K} \mathcal{K}^{\dagger}(S, T)\right)_{01,01}$

$$
=\frac{\text { Num }}{(y+2+S+T) ! !(y-S-T) ! !(y+1+S-T) ! !(y+1-S+T) ! !}
$$

with

$$
\begin{aligned}
\text { Num } & =\frac{(y+1)}{(y+3}(y+4) ! !(y-2) ! !(y+1) ! !(y+1) ! ! & & \text { for } y=\text { even } \\
& =(y+2) ! ! y ! !(y+3) ! !(y-1) ! ! & & \text { for } y=\text { odd }
\end{aligned}
$$

while $\left(\mathcal{K} \mathcal{K}^{\dagger}(S, T)\right)_{10,01}=0$. Note, however, that $K_{S} K_{T}=01$ does not exist for $T=0$ so that the $\mathcal{K} \mathcal{K}^{\dagger}$ matrix becomes one-dimensional with $\mathcal{K} \mathcal{K}_{10,10}^{\dagger}$ given by the above. (Similarly for $S=0$.) 
For $[y+1 y 10]$, with $y+S+T=$ odd:

$\left(\mathcal{K K}^{\dagger}(S, T)\right)_{10,10}=[(y+1)(y+2)(y+3)-(y+3) S(S+1)-(y+1) T(T+1)] \mathrm{CF}$

$\left(\mathcal{K} \mathcal{K}^{\dagger}(S, T)\right)_{01,01}=[(y+1)(y+2)(y+3)-(y+1) S(S+1)-(y+3) T(T+1)] \mathrm{CF}$

$\left(\mathcal{K} \mathcal{K}^{\dagger}(S, T)\right)_{10,01}=2(y+2)[S(S+1) T(T+1)]^{1 / 2} \mathrm{CF}$

with

$\mathrm{CF}=\frac{\text { Num }}{(y+3+S+T) ! !(y+1-S-T) ! !(y+2+S-T) ! !(y+2-S+T) ! !}$

Num $=\frac{1}{(y+3)}(y+4) ! !(y-2) ! !(y+1) ! !(y+1) ! ! \quad$ for $y=$ even

$$
=\frac{1}{(y+1)}(y+2) ! ! y ! !(y+3) ! !(y-1) ! ! \quad \text { for } y=\text { odd. }
$$

Note that this $2 \times 2$ matrix has one zero eigenvalue for the special case of maximum possible $S+T$ value, $S+T=y+1$, so that there is only a single physically allowed state of this type.

The next case (with $a=3$ ) contains the irreps $[y+3$ y00] and [yy30] with twodimensional $\mathcal{K} \mathcal{K}^{\dagger}$ matrices:

For $[y+3 y 00] \quad$ with $y+S+T=$ even (upper sign)

and with $y+S+T=$ odd (lower sign)

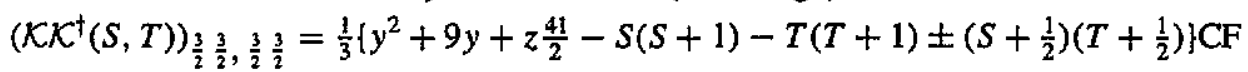

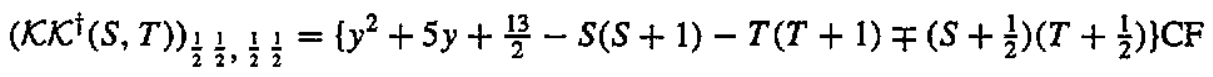

$\left(\mathcal{K} \mathcal{K}^{\dagger}(S, T)\right)_{\frac{3}{2}} \frac{3}{2}, \frac{1}{2} \frac{1}{2}=-\frac{1}{4}[(2 S-1)(2 S+3)(2 T-1)(2 T+3)]^{1 / 2} \mathrm{CF}$.

For $y+S+T=$ even:

$\mathrm{CF}=\frac{\text { Num }}{(y+4+S+T) ! !(y+2-S-T) ! !(y+4+S-T) ! !(y+4-S+T) ! !}$

while for $y+S+T=$ odd:

$\mathrm{CF}=\frac{\text { Num }}{(y+5+S+T) ! !(y+3-S-T) ! !(y+3+S-T) ! !(y+3-S+T) ! !}$

with

$$
\begin{aligned}
\text { Num } & =(y+6) ! ! y ! !(y+1) ! !(y+3) ! ! & & \text { for } y=\text { even } \\
& =(y+4) ! !(y+2) ! !(y+5) ! !(y-1) ! ! & & \text { for } y=\text { odd }
\end{aligned}
$$

for both cases.

Equations (54) can also be used to give the $\mathcal{K} \mathcal{K}^{\dagger}$ matrices for the conjugate irrep $[y+3 y+330]$ if $K_{S} K_{T}=\frac{3}{2} \frac{3}{2}$ and $\frac{1}{2} \frac{1}{2}$ are replaced by $K_{S} K_{T}=\frac{3}{2}-\frac{3}{2}$ and $\frac{1}{2}-\frac{1}{2}$.

Irreps $[y+2 y$ 10] and [ $y+1 \quad y 20]$, and similarly irreps with $a=4$ starting with [y+4 y 00], contain $S, T$ values with $\mathcal{K} \mathcal{K}^{\dagger}$ matrices of dimension 3 . Such matrices can best be calculated numerically for any $S T$ value by the recursive process used to calculate the general alnalytic expressions given by equations (47)-(54). There are, however, a number of irreps with purely one-dimensional $\mathcal{K} \mathcal{K}^{\dagger}$ matrices. These include the totally symmetric irreps [y000] with $S=T=\frac{y}{2}, \frac{y}{2}-1, \ldots, 0$, or $\frac{1}{2}$. The reduced matrix elements of equations (33) and 
(34) at once lead to:

For [y000]; or for [yyy0]; with $T=S$ :

$$
\begin{array}{ll}
\mathcal{K K}^{\dagger}(S, S)=\frac{\text { Num }}{(y-2 S) ! !(y+2 S+2) ! !} & \\
\begin{array}{rlrl}
\text { Num } & =y ! !(y+2) ! ! & & \text { for } y=\text { even } \\
& =(y-1) ! !(y+3) ! ! & \text { for } y=\text { odd. }
\end{array}
\end{array}
$$

In addition, irreps [y100] with $T=S$, or $T=S \pm 1$, up to maximum $S, T=$ $\frac{1}{2}(y+1), \frac{1}{2}(y-1)$ or $\frac{1}{2}(y-1), \frac{1}{2}(y+1)$ have one-dimensional $\mathcal{K} \mathcal{K}^{\dagger}$ matrices completely specified by $S$ and $T$. The vCS state vectors for these irreps can be constructed by repeated action with the generators, $E$, on the starting states with $S, T=\frac{1}{2} \frac{1}{2}$ for $y+1=$ odd or $S, T=10$ for $y+1=$ even. We obtain the (normalized) states

$$
\begin{aligned}
\Psi_{S M_{S}, T=S M_{T}}^{[y 100]}(\Omega) & =\left[\frac{3}{S(S+1)(2 S+1)}\right]^{1 / 2} \sum_{K=-S}^{+S} K(-1)^{K-(y+1) / 2} \\
\times & D_{K M_{S}}^{S}\left(\Omega_{S}\right) D_{K M_{T}}^{T=S}\left(\Omega_{T}\right) \frac{(2 S+1)}{8 \pi^{2}} \\
\Psi_{S M_{S}, S+1, M_{T}}^{[y 100]}(\Omega) & =\left[\frac{3}{(S+1)(2 S+1)(2 S+3)}\right]^{1 / 2} \sum_{K=-S}^{+S}\left[(S+1)^{2}-K^{2}\right]^{1 / 2}(-1)^{K-(y+1) / 2} \\
\times & \times D_{K M_{S}}^{S}\left(\Omega_{S}\right) D_{K M_{T}}^{T=S+1}\left(\Omega_{T}\right) \frac{[(2 S+1)(2 S+3)]^{1 / 2}}{8 \pi^{2}}
\end{aligned}
$$

with analagous expression for $S, T=S+1, S$. (Similar expansions are obtained for the conjugate irreps, [yyy-10], if the $K_{T}$ values $K_{T}=K_{S}$ are replaced by $K_{T}=-K_{S}$ and the phase factor $(-1)^{K-(y+1) / 2}$ is replaced by +1 .)

With equations (22)-(24), these lead to the reduced matrix elements

$$
\begin{aligned}
& \langle[y 100] S+1 S\|\Gamma(E)\|[y 100] S S\rangle=\frac{[(2 S+1)(2 S+3)]^{1 / 2}}{2(S+1)}(y+1) \\
& \langle[y 100] S S\|\Gamma(E)\|[y 100] S+1 S\rangle=\frac{-[(2 S+1)(2 S+3)]^{1 / 2}}{2(S+1)}(y+3+2 S) \\
& \langle[y 100] S+1 S+1\|\Gamma(E)\|[y 100] S S\rangle=\frac{[S(S+2)(2 S+1)(2 S+3)]^{1 / 2}}{2(S+1)}(y-1-2 S) \\
& \langle[y 100] S S\|\Gamma(E)\|[y 100] S+1 S+1\rangle=\frac{[S(S+2)(2 S+1)(2 S+3)]^{1 / 2}}{2(S+1)}(y+3+2 S) .
\end{aligned}
$$

For $[y 100]$ the $\mathcal{K} \mathcal{K}^{\dagger}$ values are

$$
\begin{aligned}
& \mathcal{K} \mathcal{K}^{\dagger}(S, S)=\frac{\mathrm{Num}}{(y+1+2 S) ! !(y-1-2 S) ! !} \\
& \mathrm{Num}= \begin{cases}(y+2) ! !(y-2) ! ! & \text { for } y=\text { even } \\
\frac{1}{(y+1)}(y-1) ! !(y+3) ! ! & \text { for } y=\text { odd }\end{cases} \\
& \begin{aligned}
\mathcal{K} \mathcal{K}^{\dagger}(S+1, S)=\mathcal{K} \mathcal{K}^{\dagger}(S, S+1) \\
=\frac{y+1}{(y-1-2 S)} \mathcal{K} \mathcal{K}^{\dagger}(S+1, S+1) .
\end{aligned}
\end{aligned}
$$

Identical $\mathcal{K} \mathcal{K}^{\dagger}$ values are obtained for the conjugate [yy $\left.y-10\right]$. 
In addition, the irrep [ $y 110]$, again with $T=S$ or $T=S \pm 1$, with maximum $S, T=\frac{y}{2} \frac{y}{2}$, again has only one-dimensional $\mathcal{K} K^{\dagger}$ matrices. In this case

$$
\begin{aligned}
\Psi_{S M_{S}, T=S M_{T}}^{(y 110]}(\Omega) & =\left[\frac{3}{4 S(S+1)(2 S+1)}\right]^{1 / 2} \sum_{K=-S}^{S}[(S+K)(S+1-K)]^{1 / 2}(-1)^{K-\frac{y}{2}} \\
& \times\left\{D_{K M_{S}}^{S}\left(\Omega_{S}\right) D_{K-1 M_{T}}^{S}\left(\Omega_{T}\right)+(-1)^{2 S+1} D_{-K M_{S}}^{S}\left(\Omega_{S}\right) D_{-(K-1) M_{T}}^{S}\left(\Omega_{T}\right)\right\}
\end{aligned}
$$

and

$$
\begin{aligned}
& \Psi_{S+1 M_{S}, T=S M_{T}}^{[\mathrm{y} 110 \mathrm{f}}(\Omega)=\left[\frac{3}{4(S+1)(2 S+1)(2 S+3)}\right]^{1 / 2} \\
& \times \sum_{K=-(S+1)}^{S+1}[(S+K)(S+1+K)]^{1 / 2}(-1)^{K-\frac{y}{2}} \\
& \times\left\{D_{K M_{S}}^{S+1}\left(\Omega_{S}\right) D_{K-1 M_{T}}^{S}\left(\Omega_{T}\right)+(-1)^{2 S} D_{-K M_{S}}^{S+1}\left(\Omega_{S}\right) D_{-(K-1) M_{T}}^{S}\left(\Omega_{T}\right)\right\} \\
& \Psi_{S M_{S}, T=S+1 M_{T}}^{[y: 110]}(\Omega)=\left[\frac{3}{4(S+1)(2 S+1)(2 S+3)}\right]^{1 / 2} \\
& \times \sum_{K=-S}^{+S}[(S+1-K)(S+2-K)]^{1 / 2}(-1)^{K-\frac{y}{2}} \\
& \times\left\{D_{K M_{S}}^{S}\left(\Omega_{S}\right) D_{K-1 M_{T}}^{S+1}\left(\Omega_{T}\right)+(-1)^{2 S} D_{-K M_{S}}^{S}\left(\Omega_{S}\right) D_{-(K-1) M_{T}}^{S+1}\left(\Omega_{T}\right)\right\} .
\end{aligned}
$$

These lead to the reduced matrix elements

$\langle[y 110] S+1 S+1\|\Gamma(E)\|[y 110] S S\rangle=\frac{[S(S+2)(2 S+1)(2 S+3)]^{1 / 2}}{2(S+1)}(y-2 S)$
$\langle[y 110] S S\|\Gamma(E)\|[y 110] S+1 S+1\rangle=\frac{[S(S+2)(2 S+1)(2 S+3)]^{1 / 2}}{2(S+1)}(y+4+2 S)$

$\langle[y 110] S S-1|| \Gamma(E)||[y 110] S S\rangle=\frac{-[(2 S-1)(2 S+1)]^{1 / 2}}{2 S}(y+2+2 S)$,

$\langle[y 110] S S\|\Gamma(E)\|[y 110] S S-1\rangle=\frac{[(2 S-1)(2 S+1)]^{1 / 2}}{2 S}(y+2)$.

The recursion formulae then give the $\mathcal{K} \mathcal{K}^{\dagger}$ values.

For $[y 110]$ :

$$
\begin{aligned}
& \operatorname{KK}^{\dagger}(S, S)=\frac{\text { Num }}{(y-2 S) ! !(y+2+2 S) ! !} \\
& \begin{aligned}
\text { Num }=(y-2) ! !(y+2) ! !(y+2) & \text { for } y=\text { even } \\
=(y-1) ! !(y+3) ! ! & \text { for } y=\text { odd }
\end{aligned} \\
& \begin{array}{rlr}
\mathcal{K K}^{\dagger}(S-1, S) & =\mathcal{K} \mathcal{K}^{\dagger}(S, S-1) & \\
& =\frac{(y+2+2 S)}{(y+2)} \mathcal{K} \mathcal{K}^{\dagger}(S, S) .
\end{array}
\end{aligned}
$$

The conjugate irrep $[y y-1 y-10]$ again has the same $\mathcal{K} \mathcal{K}^{\dagger}$ values.

An alternative method for finding the $\mathcal{K} \mathcal{K}^{\dagger}$ matrices would start the calculational process with the states of maximum $(S+T)$ values, $S+T=f_{1}-f_{4}$. For such states equation (41) leads to a set of linear equations in the $\mathcal{K} \mathcal{K}^{\dagger}$ matrix elements for maximum $(S+T)$ values 
if $S^{\prime}, T^{\prime}$ are chosen such that $S^{\prime}+T^{\prime}>f_{\mathrm{t}}-f_{4}$, so that the left-hand side of equation (41) is zero. As a specific example, the $\mathcal{K} \mathcal{K}^{\dagger}$ matrix for the irrep $[y+2$ y 10$]$ for $S, T$ values such that $S+T=y+2$ is given (to within a common factor $C=C(y, S)$ ) by

$$
\left(\begin{array}{ccc}
\left(S+\frac{3}{2}\right)\left(T-\frac{1}{2}\right) C & -2\left(T-\frac{1}{2}\right) \sqrt{\left(S-\frac{1}{2}\right)\left(S+\frac{3}{2}\right) C} & \sqrt{\left(S-\frac{1}{2}\right)\left(S+\frac{3}{2}\right)\left(T-\frac{1}{2}\right)\left(T+\frac{3}{2}\right)} C \\
-2\left(T-\frac{1}{2}\right) \sqrt{\left(S-\frac{1}{2}\right)\left(S+\frac{3}{2}\right) C} & 4\left(S-\frac{1}{2}\right)\left(T-\frac{1}{2}\right) C & -2\left(S-\frac{1}{2}\right) \sqrt{\left(T-\frac{1}{2}\right)\left(T+\frac{3}{2}\right) C} \\
\sqrt{\left(S-\frac{1}{2}\right)\left(S+\frac{3}{2}\right)\left(T-\frac{1}{2}\right)\left(T+\frac{3}{2}\right) C} & -2\left(S-\frac{1}{2}\right) \sqrt{\left(T-\frac{1}{2}\right)\left(T+\frac{3}{2}\right) C} & \left(S-\frac{1}{2}\right)\left(T+\frac{3}{2}\right) C
\end{array}\right)
$$

with rows and columns $K_{S} K_{T}=\frac{3}{2} \frac{1}{2}, \frac{1}{2}-\frac{1}{2}, \frac{1}{2} \frac{3}{2}$, in that order. The only non-zero eigenvalue of this matrix leads to the single physically allowed state with index $i=1$ (see equations (43), (44)) and

$$
\begin{aligned}
& U_{i=1, \frac{3}{2} \frac{1}{2}}=\sqrt{\frac{\left(S+\frac{3}{2}\right)\left(T-\frac{1}{2}\right)}{F(y, S)}} \\
& U_{i=1, \frac{1}{2}-\frac{1}{2}}=-2 \sqrt{\frac{\left(S-\frac{1}{2}\right)\left(T-\frac{1}{2}\right)}{F(y, S)}} \\
& U_{i=1, \frac{1}{2} \frac{3}{2}}=\sqrt{\frac{\left(S-\frac{1}{2}\right)\left(T+\frac{3}{2}\right)}{F(y, S)}}
\end{aligned}
$$

with $F(y, S)=\left(S+\frac{3}{2}\right)\left(T-\frac{1}{2}\right)+4\left(S-\frac{1}{2}\right)\left(T-\frac{1}{2}\right)+\left(S-\frac{1}{2}\right)\left(T+\frac{3}{2}\right)$, where $T=y+2-S$. The common factor $C(y, S)$ can be evaluated (to within a constant) by requiring the Hermiticity of the matrix elements for the single allowed states; e.g.

$$
\langle i=1 ; S+1 T-1\|E\| i=1 ; S T\rangle=\langle i=1 ; S T\|E\| i=1 ; S+1 T-1\rangle
$$

(see equation (45)). This leads to the recursive formula

$$
\frac{C(y, S+1)}{C(y, S)}=\frac{S+1}{y+2-S}
$$

or

$$
C(y, S)=\frac{(2 S) ! !(2 y+4-2 S) ! !}{3(2 y+1) ! !} C\left(y, \frac{3}{2}\right) .
$$

If needed, the single constant $C\left(y, \frac{3}{2}\right)$ can now be determined by relating the $\mathcal{K} \mathcal{K}^{\dagger}$ matrix elements for maximum $(S+T)$ values to the starting matrix $\mathcal{K} \mathcal{K}^{\dagger}\left(\frac{1}{2}, \frac{1}{2}\right)=1$. However, the reduced matrix elements of $E$ are independent of the constant $C\left(y, \frac{3}{2}\right)$ (cf equation (45)).

\section{Summary}

A double rotor coherent state expansion for the states of the SU(4) $\supset \mathrm{SU}(2) \times \mathrm{SU}(2)$ Wigner supermultiplet scheme leads to a very simple matrix representation of this algebra. Matrix elements of the vCS realizations for the generators $S, T, E$ are given by very simple expressions, involving ordinary angular momentum spin and isospin Wigner coefficients with projection (intrinsic) quantum numbers $K_{S}, K_{T}$ (see, in particular, equations (22)(27)). The $\mathcal{K}$-matrix technique of vCS theory is used to convert these non-Hermitian matrices to Hermitian form (see, in particular, equation (45)). The $\mathcal{K}$ matrices convert the infinite-dimensional double-rotor space to the physical subspace of a given SU(4) irreducible representation. $\mathcal{K}$-matrix theory thus effectively elevates the labels $K_{S}, K_{T}$ to the role of good quantum numbers. In view of the central role of the $\mathcal{K}$ matrices, analytic expressions 
are given for $\mathcal{K} \mathcal{K}^{\dagger}$ (as functions of $S, T$, and SU(4) irrep labels) for all SU(4) irreps which lead to $\mathcal{K} \mathcal{K}^{\dagger}$ matrices with dimension $\leqslant 2$. These include the $U(4)$ representations [yy00], $[y \quad y-100],[y+2 y 00],[y+1$ y 10$]],[y+3 y 00],[y 000],[y 100],[y 110]$ and their conjugates. Effectively, this gives analytic expressions for the $\mathrm{SU}(4) \supset \mathrm{SU}(2) \times \mathrm{SU}(2)$ algebra for most of the irreps of interest in actual applications of the supermultiplet scheme. The only numerical work required involves the determination of the $U$ matrices which diagonalize $\mathcal{K} \mathcal{K}^{\dagger}$ in the case of multidimensional $\mathcal{K} \mathcal{K}^{\dagger}$ matrices. The double-rotor vCs expansion of this investigation can be combined with the techniques of [7] and [11] to calculate the most general Wigner coefficients in the $S U(4) \supset S U(2) \times S U(2)$ Wigner supermultiplet scheme. The details will be given in a future contribution.

\section{Appendix. Rotation group conventions}

The $R\left(\Omega_{S}\right), R\left(\Omega_{T}\right)$ operators of this investigation follow the conventions of [4]. Since these reverse the role of the quantum numbers $K$ and $M$ in comparison with the more frequently used nuclear physics conventions [10], a careful definition is given here. The $D_{K M}^{J}(\Omega)$ of this investigation (with $J=S$ or $T$, for $\Omega=\Omega_{S}$ or $\Omega_{T}$ ) are given by $\mathrm{e}^{\mathrm{i} K \alpha} d_{K M}^{J}(\beta) \mathrm{e}^{\mathrm{i} M \gamma}$ with $d_{K M}^{J}(\beta)=\left\langle J K\left|\mathrm{e}^{+\mathrm{i} \beta J y}\right| J M\right\rangle$. This leads to the vCS realizations (cf equations (11) and (12))

$$
\Gamma\left(J_{0}\right)=\frac{1}{\mathrm{i}} \frac{\partial}{\partial \gamma} \quad \Gamma\left(J_{ \pm}\right)=\mathrm{e}^{ \pm \mathrm{i} \gamma}\left\{\mathrm{i} \cot \beta \frac{\partial}{\partial \gamma} \pm \frac{\partial}{\partial \beta}\right\} .
$$

On the other hand, the left vcs realizations or intrinsic operators $\bar{J}$ (cf equations (13), (15)), are given by

$$
\bar{\Gamma}\left(J_{0}\right)=\frac{1}{\mathrm{i}} \frac{\partial}{\partial \alpha} \quad \bar{\Gamma}\left(J_{ \pm}\right)=\mathrm{e}^{\mp \mathrm{i} \alpha}\left\{\frac{\mathrm{i}}{\sin \beta} \frac{\partial}{\partial \gamma}-\mathrm{i} \cot \beta \frac{\partial}{\partial \alpha} \pm \frac{\partial}{\partial \beta}\right\} .
$$

Note added in proof. A recent article on 'Vector coherent state representations of so(5) in an so(3) basis' by D J Rowe is to be published in J. Math. Phys.

\section{References}

[1] Rowe D J 1984 J. Math. Phys. 252662

Rowe D J, Rosensteel G and Gilmore R 1985 J. Math. Phys. 262787

LeBlane R and Rowe D J 1985 J. Phys. A: Math. Gen. 18 1891, 1905; 1986 J. Phys. A: Math. Gen. 191083

[2] Deenen J and Quesne C 1984 J. Math. Phys. 25 1638, 2354

Quesne C 1986 J. Math. Phys. 27869

[3] Hecht K T 1987 The vector coherent state method and its applications to problems of higher symmetry (Springer Lecture Notes in Physics 290) (New York: Springer)

[4] Rowe D J, LeBlanc R and Repka J 1989 J. Phys. A: Math. Gen. 22 L309

[5] Elliott J P 1958 Proc. Roy. Soc. A 245 128, 562

[6] Rowe D J 1994 Proc. Contemporary Physics (Drexel University, 1991) (Singapore: World Scientific) (to be published)

[7] Hecht K T 1990 J. Phys. A: Math. Gen. 23407

[8] Draayer J P 1970 J. Math. Phys. 113225

[9] Hecht K T and Sing Chin Pang 1969 J. Math. Phys. 101571

[10] Bohr A and Mottelson B R 1969 Nuclear Structure (New York: Benjamin)

[11] LeBlanc R, Hecht K T and Biedenharn L C 1991 J. Phys. A: Math. Gen. 241393

Hecht K T 1993 J. Phys. A: Math. Gen. 26329 